\title{
Measurement of the Branching Fractions of $\bar{B} \rightarrow D^{* *} \ell^{-} \bar{\nu}_{\ell}$ Decays in Events Tagged by a Fully Reconstructed $B$ Meson
}

B. Aubert, ${ }^{1}$ M. Bona, ${ }^{1}$ Y. Karyotakis,${ }^{1}$ J. P. Lees,${ }^{1}$ V. Poireau, ${ }^{1}$ E. Prencipe,${ }^{1}$ X. Prudent,${ }^{1}$ V. Tisserand,${ }^{1}$ J. Garra Tico, ${ }^{2}$ E. Grauges, ${ }^{2}$ L. Lopez,${ }^{3 a, 3 b}$ A. Palano, ${ }^{3 a, 3 b}$ M. Pappagallo, ${ }^{3 a, 3 b}$ G. Eigen, ${ }^{4}$ B. Stugu, ${ }^{4}$ L. Sun, ${ }^{4}$ G. S. Abrams, ${ }^{5}$ M. Battaglia, ${ }^{5}$

D. N. Brown, ${ }^{5}$ R. N. Cahn, ${ }^{5}$ R. G. Jacobsen, ${ }^{5}$ L. T. Kerth, ${ }^{4}$ Yu. G. Kolomensky, ${ }^{5}$ G. Lynch,${ }^{5}$ I. L. Osipenkov, ${ }^{5}$

M. T. Ronan, ${ }^{5, *}$ K. Tackmann, ${ }^{5}$ T. Tanabe, ${ }^{5}$ C. M. Hawkes, ${ }^{6}$ N. Soni, ${ }^{6}$ A. T. Watson, ${ }^{6}$ H. Koch, ${ }^{7}$ T. Schroeder, ${ }^{7}$ D. Walker, ${ }^{8}$ D. J. Asgeirsson, ${ }^{9}$ B. G. Fulsom, ${ }^{9}$ C. Hearty, ${ }^{9}$ T. S. Mattison, ${ }^{9}$ J. A. McKenna, ${ }^{9}$ M. Barrett, ${ }^{10}$ A. Khan, ${ }^{10}$ V. E. Blinov, ${ }^{11}$ A. D. Bukin, ${ }^{11}$ A. R. Buzykaev, ${ }^{11}$ V.P. Druzhinin, ${ }^{11}$ V. B. Golubev, ${ }^{11}$ A. P. Onuchin, ${ }^{11}$ S. I. Serednyakov, ${ }^{11}$

Yu. I. Skovpen, ${ }^{11}$ E. P. Solodov, ${ }^{11}$ K. Yu. Todyshev, ${ }^{11}$ M. Bondioli, ${ }^{12}$ S. Curry, ${ }^{12}$ I. Eschrich,${ }^{12}$ D. Kirkby, ${ }^{12}$ A. J. Lankford, ${ }^{12}$ P. Lund, ${ }^{12}$ M. Mandelkern, ${ }^{12}$ E. C. Martin, ${ }^{12}$ D. P. Stoker, ${ }^{12}$ S. Abachi, ${ }^{13}$ C. Buchanan, ${ }^{13}$ J. W. Gary, ${ }^{14}$ F. Liu, ${ }^{14}$ O. Long, ${ }^{14}$ B. C. Shen, ${ }^{14}$,* G. M. Vitug, ${ }^{14}$ Z. Yasin, ${ }^{14}$ L. Zhang, ${ }^{14}$ V. Sharma, ${ }^{15}$ C. Campagnari, ${ }^{16}$ T. M. Hong, ${ }^{16}$

D. Kovalskyi, ${ }^{16}$ M. A. Mazur, ${ }^{16}$ J. D. Richman, ${ }^{16}$ T. W. Beck, ${ }^{17}$ A. M. Eisner, ${ }^{17}$ C. J. Flacco,${ }^{17}$ C. A. Heusch,${ }^{17}$

J. Kroseberg, ${ }^{17}$ W. S. Lockman, ${ }^{17}$ T. Schalk, ${ }^{17}$ B. A. Schumm, ${ }^{17}$ A. Seiden, ${ }^{17}$ L. Wang, ${ }^{17}$ M. G. Wilson, ${ }^{17}$

L. O. Winstrom, ${ }^{17}$ C. H. Cheng, ${ }^{18}$ D. A. Doll ${ }^{18}$ B. Echenard,${ }^{18}$ F. Fang, ${ }^{18}$ D. G. Hitlin, ${ }^{18}$ I. Narsky, ${ }^{18}$ T. Piatenko, ${ }^{18}$

F. C. Porter ${ }^{18}$ R. Andreassen, ${ }^{19}$ G. Mancinelli, ${ }^{19}$ B. T. Meadows, ${ }^{19}$ K. Mishra, ${ }^{19}$ M. D. Sokoloff, ${ }^{19}$ P. C. Bloom, ${ }^{20}$

W. T. Ford, ${ }^{20}$ A. Gaz,${ }^{20}$ J. F. Hirschauer, ${ }^{20}$ M. Nagel, ${ }^{20}$ U. Nauenberg, ${ }^{20}$ J. G. Smith, ${ }^{20}$ K. A. Ulmer, ${ }^{20}$ S. R. Wagner ${ }^{20}$

R. Ayad,${ }^{20}$ A. Soffer, ${ }^{21, \dagger}$ W. H. Toki, ${ }^{21+}$ R. J. Wilson, ${ }^{21}$ D. D. Altenburg, ${ }^{22}$ E. Feltresi, ${ }^{22}$ A. Hauke, ${ }^{22}$ H. Jasper,${ }^{22}$

M. Karbach, ${ }^{22}$ J. Merkel, ${ }^{22}$ A. Petzold, ${ }^{22}$ B. Spaan, ${ }^{22}$ K. Wacker, ${ }^{22}$ M. J. Kobel, ${ }^{23}$ W. F. Mader, ${ }^{23}$ R. Nogowski, ${ }^{23}$

K. R. Schubert, ${ }^{23}$ R. Schwierz, ${ }^{23}$ J.E. Sundermann, ${ }^{23}$ A. Volk, ${ }^{23}$ D. Bernard,${ }^{24}$ G. R. Bonneaud, ${ }^{24}$ E. Latour, ${ }^{24}$

Ch. Thiebaux, ${ }^{24}$ M. Verderi, ${ }^{24}$ P. J. Clark, ${ }^{25}$ W. Gradl, ${ }^{25}$ S. Playfer, ${ }^{25}$ J. E. Watson, ${ }^{25}$ M. Andreotti, ${ }^{26 a, 26 b}$ D. Bettoni, ${ }^{26 a}$

C. Bozzi, ${ }^{26 a}$ R. Calabrese, ${ }^{26 a, 26 b}$ A. Cecchi, ${ }^{26 a, 26 b}$ G. Cibinetto, ${ }^{26 a, 26 b}$ P. Franchini ${ }^{26 a, 26 b}$ E. Luppi, ${ }^{26 a, 26 b}$ M. Negrini, ${ }^{26 a, 26 b}$

A. Petrella, ${ }^{26 a, 26 b}$ L. Piemontese, ${ }^{26 a}$ V. Santoro, ${ }^{26 a, 26 b}$ R. Baldini-Ferroli, ${ }^{27}$ A. Calcaterra, ${ }^{27}$ R. de Sangro, ${ }^{27}$

G. Finocchiaro, ${ }^{27}$ S. Pacetti, ${ }^{27}$ P. Patteri, ${ }^{27}$ I. M. Peruzzi,${ }^{27,8}$ M. Piccolo,${ }^{27}$ M. Rama, ${ }^{27}$ A. Zallo, ${ }^{27}$ A. Buzzo, ${ }^{28 a}$

R. Contri, ${ }^{28 a, 28 b}$ M. Lo Vetere, ${ }^{28 a, 28 b}$ M. M. Macri, ${ }^{28 a}$ M. R. Monge, ${ }^{28 a, 28 b}$ S. Passaggio, ${ }^{28 a}$ C. Patrignani, ${ }^{28 a, 28 b}$

E. Robutti, ${ }^{28 \mathrm{a}}$ A. Santroni, ${ }^{28 \mathrm{a}, 28 \mathrm{~b}}$ S. Tosi, ${ }^{28 \mathrm{a}, 28 \mathrm{~b}}$ K. S. Chaisanguanthum, ${ }^{29}$ M. Morii, ${ }^{29}$ J. Marks, ${ }^{30}$ S. Schenk, ${ }^{30}$ U. Uwer, ${ }^{30}$

V. Klose ${ }^{31}$ H. M. Lacker, ${ }^{31}$ D. J. Bard, ${ }^{32}$ P. D. Dauncey, ${ }^{32}$ J. A. Nash, ${ }^{32}$ W. Panduro Vazquez, ${ }^{32}$ M. Tibbetts, ${ }^{32}$

P. K. Behera, ${ }^{33}$ X. Chai, ${ }^{33}$ M. J. Charles, ${ }^{33}$ U. Mallik,,${ }^{33}$ J. Cochran, ${ }^{34}$ H. B. Crawley, ${ }^{34}$ L. Dong, ${ }^{34}$ W. T. Meyer, ${ }^{34}$ S. Prell, ${ }^{34}$

E. I. Rosenberg, ${ }^{34}$ A. E. Rubin, ${ }^{34}$ Y. Y. Gao, ${ }^{35}$ A. V. Gritsan, ${ }^{35}$ Z. J. Guo,${ }^{35}$ C. K. Lae, ${ }^{35}$ A. G. Denig, ${ }^{36}$ M. Fritsch,${ }^{36}$

G. Schott,${ }^{36}$ N. Arnaud,${ }^{37}$ J. Béquilleux,${ }^{37}$ A. D'Orazio, ${ }^{37}$ M. Davier,${ }^{37}$ J. Firmino da Costa, ${ }^{37}$ G. Grosdidier,${ }^{37}$

A. Höcker, ${ }^{37}$ V. Lepeltier, ${ }^{37}$ F. Le Diberder, ${ }^{37}$ A. M. Lutz, ${ }^{37}$ S. Pruvot, ${ }^{37}$ P. Roudeau, ${ }^{37}$ M. H. Schune, ${ }^{37}$ J. Serrano, ${ }^{37}$

V. Sordini ${ }^{37, \|}$ A. Stocchi, ${ }^{37}$ G. Wormser,${ }^{37}$ D. J. Lange, ${ }^{38}$ D. M. Wright, ${ }^{38}$ I. Bingham, ${ }^{39}$ J. P. Burke, ${ }^{39}$ C. A. Chavez,${ }^{39}$

J. R. Fry, ${ }^{39}$ E. Gabathuler, ${ }^{39}$ R. Gamet, ${ }^{39}$ D. E. Hutchcroft, ${ }^{39}$ D. J. Payne, ${ }^{39}$ C. Touramanis, ${ }^{39}$ A. J. Bevan, ${ }^{40}$ C. K. Clarke, ${ }^{40}$

K. A. George,${ }^{40}$ F. Di Lodovico, ${ }^{40}$ R. Sacco,${ }^{40}$ M. Sigamani, ${ }^{40}$ G. Cowan,${ }^{41}$ H. U. Flaecher, ${ }^{41}$ D. A. Hopkins,${ }^{41}$

S. Paramesvaran, ${ }^{41}$ F. Salvatore, ${ }^{41}$ A. C. Wren, ${ }^{41}$ D. N. Brown, ${ }^{42}$ C. L. Davis, ${ }^{42}$ K. E. Alwyn, ${ }^{43}$ D. Bailey,${ }^{43}$ R. J. Barlow, ${ }^{43}$ Y. M. Chia, ${ }^{43}$ C. L. Edgar, ${ }^{43}$ G. Jackson, ${ }^{43}$ G. D. Lafferty, ${ }^{43}$ T. J. West, ${ }^{43}$ J. I. Yi,${ }^{43}$ J. Anderson, ${ }^{44}$ C. Chen,${ }^{44}$ A. Jawahery,${ }^{44}$ D. A. Roberts, ${ }^{44}$ G. Simi, ${ }^{44}$ J. M. Tuggle,${ }^{44}$ C. Dallapiccola,${ }^{45}$ X. Li ${ }^{45}$ E. Salvati, ${ }^{45}$ S. Saremi,${ }^{45}$ R. Cowan,${ }^{46}$ D. Dujmic,${ }^{46}$

P. H. Fisher, ${ }^{46}$ K. Koeneke, ${ }^{46}$ G. Sciolla ${ }^{46}$ M. Spitznagel, ${ }^{46}$ F. Taylor, ${ }^{46}$ R. K. Yamamoto, ${ }^{46}$ M. Zhao,${ }^{46}$ P. M. Patel, ${ }^{47}$

S. H. Robertson, ${ }^{47}$ A. Lazzaro, ${ }^{48 a, 48 b}$ V. Lombardo, ${ }^{48 a}$ F. Palombo, ${ }^{48 a}, 48 b$ J. M. Bauer, ${ }^{49}$ L. Cremaldi, ${ }^{49}$ V. Eschenburg, ${ }^{49}$

R. Godang, ${ }^{49, \pi}$ R. Kroeger, ${ }^{49}$ D. A. Sanders, ${ }^{49}$ D. J. Summers, ${ }^{49}$ H. W. Zhao, ${ }^{49}$ M. Simard, ${ }^{50}$ P. Taras, ${ }^{50}$ F. B. Viaud, ${ }^{50}$

H. Nicholson, ${ }^{51}$ G. De Nardo, ${ }^{52 a, 52 b}$ L. Lista, ${ }^{52 a}$ D. Monorchio, ${ }^{52 a, 52 b}$ G. Onorato, ${ }^{52 a, 52 b}$ C. Sciacca, ${ }^{52 a, 52 b}$ G. Raven, ${ }^{53}$

H. L. Snoek, ${ }^{53}$ C. P. Jessop, ${ }^{54}$ K. J. Knoepfel, ${ }^{54}$ J. M. Lo Secco, ${ }^{54}$ W. F. Wang, ${ }^{54}$ G. Benelli, ${ }^{55}$ L. A. Corwin, ${ }^{55}$

K. Honscheid, ${ }^{55}$ H. Kagan, ${ }^{55}$ R. Kass, ${ }^{55}$ J. P. Morris, ${ }^{55}$ A. M. Rahimi, ${ }^{55}$ J. J. Regensburger, ${ }^{55}$ S. J. Sekula, ${ }^{55}$ Q. K. Wong, ${ }^{55}$

N. L. Blount, ${ }^{56}$ J. Brau, ${ }^{56}$ R. Frey, ${ }^{56}$ O. Igonkina, ${ }^{56}$ J. A. Kolb,${ }^{56}$ M. Lu, ${ }^{56}$ R. Rahmat,${ }^{56}$ N. B. Sinev,${ }^{56}$ D. Strom, ${ }^{56}$

J. Strube,${ }^{56}$ E. Torrence, ${ }^{56}$ G. Castelli, ${ }^{57 a, 57 b}$ N. Gagliardi, ${ }^{57 a, 57 b}$ M. Margoni, ${ }^{57 a, 57 b}$ M. Morandin, ${ }^{57 a}$ M. Posocco, ${ }^{57 a}$

M. Rotondo, ${ }^{57 a}$ F. Simonetto, ${ }^{57 a, 57 b}$ R. Stroili, ${ }^{57 a, 57 b}$ C. Voci, ${ }^{57 a, 57 b}$ P. del Amo Sanchez, ${ }^{58}$ E. Ben-Haim, ${ }^{58}$ H. Briand, ${ }^{58}$ G. Calderini, ${ }^{58}$ J. Chauveau, ${ }^{58}$ P. David,${ }^{58}$ L. Del Buono,${ }^{58}$ O. Hamon,${ }^{58}$ Ph. Leruste, ${ }^{58}$ J. Ocariz, ${ }^{58}$ A. Perez,${ }^{58} \mathrm{~J}$. Prendki,${ }^{58}$

S. Sitt, ${ }^{58}$ L. Gladney, ${ }^{59}$ M. Biasini, ${ }^{60 a, 60 b}$ R. Covarelli, ${ }^{60 a, 60 b}$ E. Manoni, ${ }^{60 a, 60 b}$ C. Angelini, ${ }^{61 a, 61 b}$ G. Batignani, ${ }^{61 a, 61 b}$

S. Bettarini, ${ }^{61 \mathrm{a}, 61 \mathrm{~b}}$ M. Carpinelli, ${ }^{61 \mathrm{a}, 61 \mathrm{~b}, * *}$ A. Cervelli, ${ }^{61 \mathrm{a}, 61 \mathrm{~b}}$ F. Forti ${ }^{61 \mathrm{a}, 61 \mathrm{~b}}$ M. A. Giorgi, ${ }^{61 \mathrm{a}, 61 \mathrm{~b}}$ A. Lusiani, ${ }^{61 \mathrm{a}, 61 \mathrm{c}}$ G. Marchiori, ${ }^{61 a, 61 b}$ M. Morganti, ${ }^{61 \mathrm{a}, 61 \mathrm{~b}}$ N. Neri, ${ }^{61 \mathrm{a}, 61 \mathrm{~b}}$ E. Paoloni, ${ }^{61 \mathrm{a}, 61 \mathrm{~b}}$ G. Rizzo,${ }^{61 \mathrm{a}, 61 \mathrm{~b}}$ J. J. Walsh, ${ }^{61 \mathrm{a}}$ D. Lopes Pegna, ${ }^{62}$ 
C. Lu, ${ }^{62}$ J. Olsen, ${ }^{62}$ A. J. S. Smith,${ }^{62}$ A. V. Telnov,${ }^{62}$ F. Anulli, ${ }^{63 a}$ E. Baracchini, ${ }^{63 a, 63 b}$ G. Cavoto, ${ }^{63 a}$ D. del Re,${ }^{63 a, 63 b}$ E. Di Marco, ${ }^{63 a, 63 b}$ R. Faccini, ${ }^{63 a, 63 b}$ F. Ferrarotto, ${ }^{63 a}$ F. Ferroni, ${ }^{63 a, 63 b}$ M. Gaspero, ${ }^{63 a, 63 b}$ P. D. Jackson, ${ }^{63 a}$ L. Li Gioi, ${ }^{63 a}$ M. A. Mazzoni, ${ }^{63 a}$ S. Morganti, ${ }^{63 a}$ G. Piredda, ${ }^{63 a}$ F. Polci, ${ }^{63 a, 63 b}$ F. Renga, ${ }^{63 a, 63 b}$ C. Voena, ${ }^{63 a}$ M. Ebert, ${ }^{64}$ T. Hartmann, ${ }^{64}$ H. Schröder, ${ }^{64}$ R. Waldi, ${ }^{64}$ T. Adye, ${ }^{65}$ B. Franek, ${ }^{65}$ E. O. Olaiya, ${ }^{65}$ F. F. Wilson ${ }^{65}$ S. Emery, ${ }^{66}$ M. Escalier, ${ }^{66}$ L. Esteve,${ }^{66}$ S. F. Ganzhur, ${ }^{66}$ G. Hamel de Monchenault, ${ }^{66}$ W. Kozanecki, ${ }^{66}$ G. Vasseur, ${ }^{66}$ Ch. Yèche, ${ }^{66}$ M. Zito, ${ }^{66}$ X. R. Chen, ${ }^{67}$ H. Liu ${ }^{67}$ W. Park, ${ }^{67}$ M. V. Purohit ${ }^{67}$ R. M. White, ${ }^{67}$ J. R. Wilson, ${ }^{67}$ M. T. Allen, ${ }^{68}$ D. Aston, ${ }^{68}$ R. Bartoldus, ${ }^{68}$ P. Bechtle, ${ }^{68}$ J. F. Benitez, ${ }^{68}$ R. Cenci, ${ }^{68}$ J. P. Coleman, ${ }^{68}$ M. R. Convery, ${ }^{68}$ J. C. Dingfelder, ${ }^{68}$ J. Dorfan, ${ }^{68}$ G. P. Dubois-Felsmann, ${ }^{68}$ W. Dunwoodie, ${ }^{68}$ R. C. Field, ${ }^{68}$ A. M. Gabareen, ${ }^{68}$ S. J. Gowdy, ${ }^{68}$ M. T. Graham, ${ }^{68}$ P. Grenier, ${ }^{68}$ C. Hast, ${ }^{68}$ W. R. Innes, ${ }^{68}$ J. Kaminski, ${ }^{68}$ M. H. Kelsey, ${ }^{68}$ H. Kim,${ }^{68}$ P. Kim, ${ }^{68}$ M. L. Kocian, ${ }^{68}$ D. W. G. S. Leith, ${ }^{68}$ S. Li,${ }^{68}$ B. Lindquist, ${ }^{68}$ S. Luitz, ${ }^{68}$ V. Luth, ${ }^{68}$ H. L. Lynch, ${ }^{68}$ D. B. MacFarlane, ${ }^{68}$ H. Marsiske, ${ }^{68}$ R. Messner, ${ }^{68}$ D. R. Muller ${ }^{68}$ H. Neal, ${ }^{68}$ S. Nelson, ${ }^{68}$ C. P. O' Grady, ${ }^{68}$ I. Ofte, ${ }^{68}$ A. Perazzo,${ }^{68}$ M. Perl, ${ }^{68}$ B. N. Ratcliff, ${ }^{68}$ A. Roodman, ${ }^{68}$ A. A. Salnikov, ${ }^{68}$ R. H. Schindler, ${ }^{68}$ J. Schwiening, ${ }^{68}$ A. Snyder, ${ }^{68}$ D. Su, ${ }^{68}$ M. K. Sullivan, ${ }^{68}$ K. Suzuki, ${ }^{68}$ S. K. Swain, ${ }^{68}$ J. M. Thompson, ${ }^{68}$ J. Va'vra, ${ }^{68}$ A. P. Wagner, ${ }^{68}$ M. Weaver, ${ }^{68}$ C. A. West, ${ }^{68}$ W. J. Wisniewski, ${ }^{68}$ M. Wittgen ${ }^{68}$ D. H. Wright, ${ }^{68}$ H. W. Wulsin, ${ }^{68}$ A. K. Yarritu, ${ }^{68}$ K. Yi,${ }^{68}$ C. C. Young, ${ }^{68}$ V. Ziegler, ${ }^{68}$ P. R. Burchat ${ }^{69}$ A. J. Edwards, ${ }^{69}$ S. A. Majewski, ${ }^{69}$ T. S. Miyashita, ${ }^{69}$ B. A. Petersen, ${ }^{69}$ L. Wilden, ${ }^{69}$ S. Ahmed, ${ }^{70}$ M. S. Alam,${ }^{70}$ J. A. Ernst ${ }^{70}$ B. Pan,${ }^{70}$ M. A. Saeed,${ }^{70}$ S. B. Zain,${ }^{70}$ S. M. Spanier, ${ }^{71}$ B. J. Wogsland, ${ }^{71}$ R. Eckmann, ${ }^{72}$ J. L. Ritchie, ${ }^{72}$ A. M. Ruland, ${ }^{72}$ C. J. Schilling,${ }^{72}$ R. F. Schwitters ${ }^{72}$ B. W. Drummond, ${ }^{73}$ J. M. Izen, ${ }^{73}$ X. C. Lou, ${ }^{73}$ F. Bianchi, ${ }^{74 a, 74 b}$ D. Gamba,${ }^{74 a, 74 b}$ M. Pelliccioni, ${ }^{74 a, 74 b}$ M. Bomben,${ }^{75 a, 75 b}$ L. Bosisio, ${ }^{75 a, 75 b}$ C. Cartaro, ${ }^{75 a, 75 b}$ G. Della Ricca, ${ }^{75 a, 75 b}$ L. Lanceri,${ }^{75 a, 75 b}$ L. Vitale, ${ }^{75 a, 75 b}$ V. Azzolini, ${ }^{76}$ N. Lopez-March, ${ }^{76}$ F. Martinez-Vidal, ${ }^{76}$ D. A. Milanes, ${ }^{76}$ A. Oyanguren, ${ }^{76}$ J. Albert, ${ }^{77}$ Sw. Banerjee, ${ }^{77}$ B. Bhuyan, ${ }^{77}$ H. H. F. Choi, ${ }^{77}$ K. Hamano, ${ }^{77}$ R. Kowalewski, ${ }^{77}$ M. J. Lewczuk, ${ }^{77}$ I. M. Nugent, ${ }^{77}$ J. M. Roney, ${ }^{77}$ R. J. Sobie, ${ }^{77}$ T. J. Gershon, ${ }^{78}$ P. F. Harrison, ${ }^{78}$ J. Ilic, ${ }^{78}$ T. E. Latham, ${ }^{78}$ G. B. Mohanty, ${ }^{78}$ H. R. Band, ${ }^{79}$ X. Chen, ${ }^{79}$ S. Dasu, ${ }^{79}$ K. T. Flood, ${ }^{79}$ Y. Pan, ${ }^{79}$ M. Pierini, ${ }^{79}$ R. Prepost, ${ }^{79}$ C. O. Vuosalo, ${ }^{79}$ and S. L. $\mathrm{Wu}^{79}$

(The BABAR Collaboration)

\footnotetext{
${ }^{1}$ Laboratoire de Physique des Particules, IN2P3/CNRS et Université de Savoie, F-74941 Annecy-Le-Vieux, France

${ }^{2}$ Universitat de Barcelona, Facultat de Fisica, Departament ECM, E-08028 Barcelona, Spain

${ }^{3 a}$ INFN Sezione di Bari, Dipartmento di Fisica, I-70126 Bari, Italy;

${ }^{3 \mathrm{~b}}$ Università di Bari, I-70126 Bari, Italy

${ }^{4}$ University of Bergen, Institute of Physics, N-5007 Bergen, Norway

${ }^{5}$ Lawrence Berkeley National Laboratory and University of California, Berkeley, California 94720, USA

${ }^{6}$ University of Birmingham, Birmingham, B15 2TT, United Kingdom

${ }^{7}$ Ruhr Universität Bochum, Institut für Experimentalphysik 1, D-44780 Bochum, Germany

${ }^{8}$ University of Bristol, Bristol BS8 1TL, United Kingdom

${ }^{9}$ University of British Columbia, Vancouver, British Columbia, V6T $1 Z 1$ Canada

${ }^{10}$ Brunel University, Uxbridge, Middlesex UB8 3PH, United Kingdom

${ }^{11}$ Budker Institute of Nuclear Physics, Novosibirsk 630090, Russia

${ }^{12}$ University of California at Irvine, Irvine, California 92697, USA

${ }^{13}$ University of California at Los Angeles, Los Angeles, California 90024, USA

${ }^{14}$ University of California at Riverside, Riverside, California 92521, USA

${ }^{15}$ University of California at San Diego, La Jolla, California 92093, USA

${ }^{16}$ University of California at Santa Barbara, Santa Barbara, California 93106, USA

${ }^{17}$ University of California at Santa Cruz, Institute for Particle Physics, Santa Cruz, California 95064, USA

${ }^{18}$ California Institute of Technology, Pasadena, California 91125, USA

${ }^{19}$ University of Cincinnati, Cincinnati, Ohio 45221, USA

${ }^{20}$ University of Colorado, Boulder, Colorado 80309, USA

${ }^{21}$ Colorado State University, Fort Collins, Colorado 80523, USA

${ }^{22}$ Technische Universität Dortmund, Fakultät Physik, D-44221 Dortmund, Germany

${ }^{23}$ Technische Universität Dresden, Institut für Kern- und Teilchenphysik, D-01062 Dresden, Germany

${ }^{24}$ Laboratoire Leprince-Ringuet, CNRS/IN2P3, Ecole Polytechnique, F-91128 Palaiseau, France

${ }^{25}$ University of Edinburgh, Edinburgh EH9 3JZ, United Kingdom

${ }^{26 a}$ INFN Sezione di Ferrara, Dipartimento di Fisica, I-44100 Ferrara, Italy

${ }^{26 \mathrm{~b}}$ Università di Ferrara, I-44100 Ferrara, Italy

${ }^{27}$ INFN Laboratori Nazionali di Frascati, I-00044 Frascati, Italy

${ }^{28 \mathrm{a}}$ INFN Sezione di Genova, Dipartimento di Fisica, I-16146 Genova, Italy

${ }^{28 \mathrm{~b}}$ Università di Genova, I-16146 Genova, Italy
} 


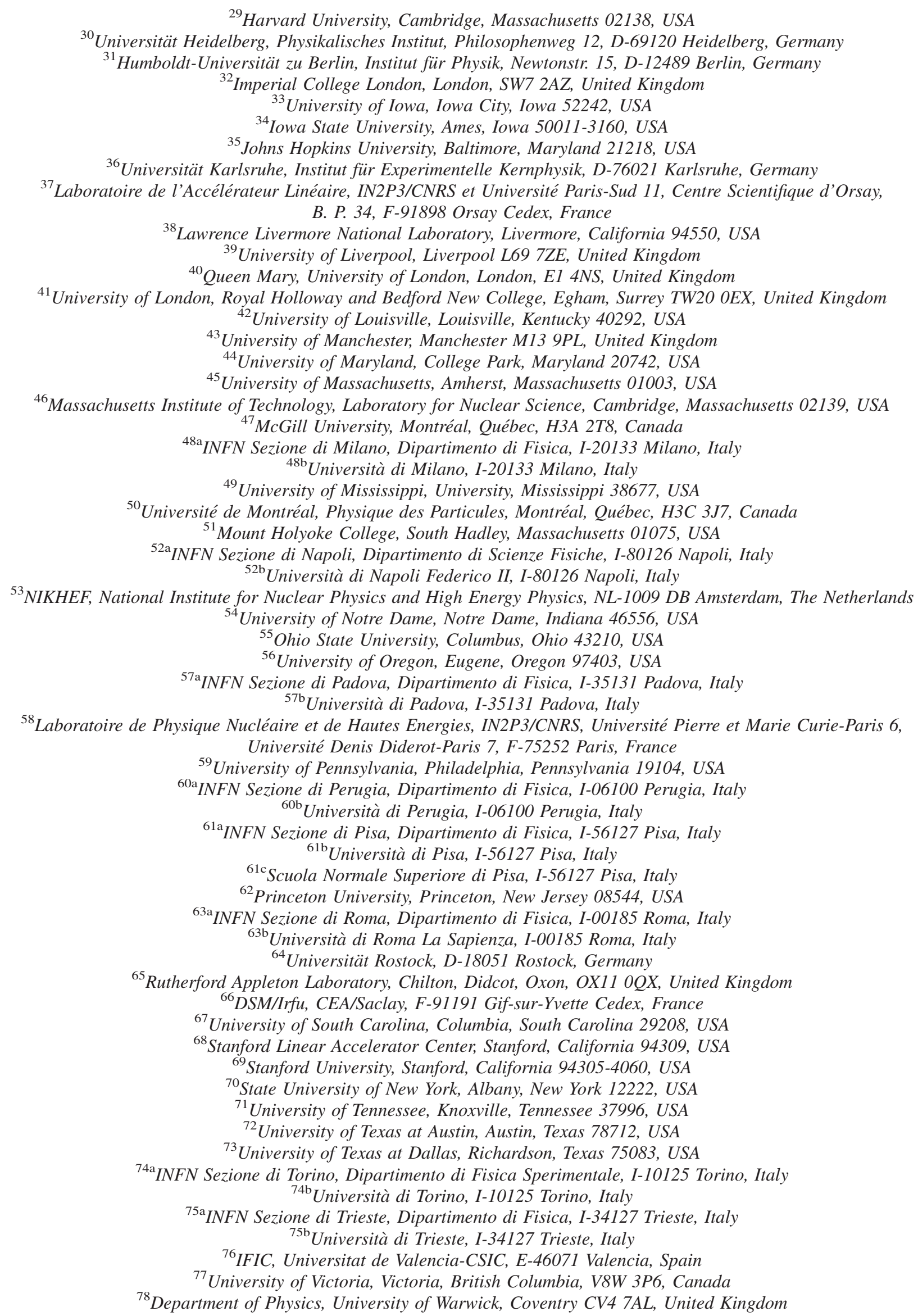


${ }^{79}$ University of Wisconsin, Madison, Wisconsin 53706, USA

(Received 4 August 2008; published 23 December 2008)

\begin{abstract}
We report a measurement of the branching fractions of $\bar{B} \rightarrow D^{* *} \ell^{-} \bar{\nu}_{\ell}$ decays based on $417 \mathrm{fb}^{-1}$ of data collected at the $Y(4 S)$ resonance with the BABAR detector at the PEP-II $e^{+} e^{-}$storage rings. Events are selected by fully reconstructing one of the $B$ mesons in a hadronic decay mode. A fit to the invariant mass differences $m\left(D^{(*)} \pi\right)-m\left(D^{(*)}\right)$ is performed to extract the signal yields of the different $D^{* *}$ states. We observe the $\bar{B} \rightarrow D^{* *} \ell^{-} \bar{\nu}_{\ell}$ decay modes corresponding to the four $D^{* *}$ states predicted by heavy quark symmetry with a significance greater than 5 standard deviations including systematic uncertainties.
\end{abstract}

DOI: 10.1103/PhysRevLett.101.261802

PACS numbers: $13.20 . \mathrm{He}, 12.38 . \mathrm{Qk}, 14.40 . \mathrm{Nd}$

Semileptonic $B$ decays to orbitally excited $P$-wave charm mesons $\left(D^{* *}\right)$ are of interest for several reasons. Improved knowledge of the branching fractions for these decays is important to reduce the systematic uncertainty in the measurements of the Cabibbo-Kobayashi-Maskawa [1] matrix elements $\left|V_{c b}\right|$ and $\left|V_{u b}\right|$. For example, one of the leading sources of systematic uncertainty on $\left|V_{c b}\right|$ measurements from $\bar{B} \rightarrow D^{*} \ell^{-} \bar{\nu}_{\ell}$ decays [2] is the limited knowledge of the background due to $\bar{B} \rightarrow D^{* *} \ell^{-} \bar{\nu}_{\ell}[3]$.

The $D^{* *}$ mesons contain one charm quark and one light quark with relative angular momentum $L=1$. According to Heavy Quark Symmetry (HQS) [4], they form one doublet of states with angular momentum $j \equiv s_{q}+L=$ $3 / 2\left[D_{1}(2420), D_{2}^{*}(2460)\right]$ and another doublet with $j=$ $1 / 2\left[D_{0}^{*}(2400), D_{1}^{\prime}(2430)\right]$, where $s_{q}$ is the light quark spin. Parity and angular momentum conservation constrain the decays allowed for each state. The $D_{1}$ and $D_{2}^{*}$ states decay through a $D$-wave to $D^{*} \pi$ and $D^{(*)} \pi$, respectively, and have small decay widths, while the $D_{0}^{*}$ and $D_{1}^{\prime}$ states decay through an $S$-wave to $D \pi$ and $D^{*} \pi$ and are very broad.

$\bar{B} \rightarrow D^{* *} \ell^{-} \bar{\nu}_{\ell}$ decays constitute a significant fraction of $B$ semileptonic decays [5] and may help to explain the discrepancy between the inclusive $\bar{B} \rightarrow X \ell^{-} \bar{\nu}_{\ell}$ rate and the sum of the measured exclusive decay rates [5-7]. The measured decay properties for $\bar{B} \rightarrow D^{* *} \ell^{-} \bar{\nu}_{\ell}$ can be compared with the predictions of the Heavy Quark Effective Theory (HQET) [8]. QCD sum rules [9] imply the strong dominance of $B$ decays to the narrow $D^{* *}$ states over those to the wide ones, while some experimental data show the opposite trend [10,11].

In this Letter, we present the observation of $B$ semileptonic decays into the four excited $D$ mesons predicted by HQS and measure the $\mathcal{B}\left(\bar{B} \rightarrow D^{* *} \ell^{-} \bar{\nu}_{\ell}\right)$ branching fractions. The analysis is based on data collected with the $B A B A R$ detector [12] at the PEP-II asymmetric-energy $e^{+} e^{-}$storage rings at SLAC. The data consist of a total of $417 \mathrm{fb}^{-1}$ recorded at the $Y(4 S)$ resonance, corresponding to approximately $460 \times 10^{6} B \bar{B}$ pairs. An additional $40 \mathrm{fb}^{-1}$, taken at a center-of-mass (c.m.) energy $40 \mathrm{MeV}$ below the $Y(4 S)$ resonance, is used to study background from $e^{+} e^{-} \rightarrow f \bar{f}(f=u, d, s, c, \tau)$ continuum events. A detailed GEANT4-based Monte Carlo (MC) simulation [13] of $B \bar{B}$ and continuum events is used to study the detector response, its acceptance, and to validate the analy- sis techniques. The simulation describes $\bar{B} \rightarrow D^{* *} \ell^{-} \bar{\nu}_{\ell}$ decays using the ISGW2 model [14] and nonresonant $\bar{B} \rightarrow$ $D^{(*)} \pi \ell^{-} \bar{\nu}_{\ell}$ decays using the model of Goity and Roberts [15].

We select semileptonic $\bar{B} \rightarrow D^{* *} \ell^{-} \bar{\nu}_{\ell}$ decays with $\ell=$ $e, \mu$ in events containing a fully reconstructed $B$ meson $\left(B_{\text {tag }}\right)$, which allows us to constrain the kinematics, reduce the combinatorial background, and determine the charge and flavor of the signal $B$ meson. $D^{* *}$ mesons are reconstructed in the $D^{(*)} \pi^{ \pm}$decay modes, and the different $D^{* *}$ states are identified by a fit to the invariant mass differences $m\left(D^{(*)} \pi\right)-m\left(D^{(*)}\right)$.

We first reconstruct the semileptonic $B$ decay, selecting a lepton with momentum $p_{\ell}^{*}$ in the c.m. frame larger than $0.6 \mathrm{GeV} / c$. We search for pairs of oppositely charged tracks that form a vertex and remove those with an invariant mass consistent with a photon conversion or a $\pi^{0}$ Dalitz decay. Candidate $D^{0}$ mesons that have the correct charge correlation with the lepton are reconstructed in the $K^{-} \pi^{+}$, $K^{-} \pi^{+} \pi^{0}, K^{-} \pi^{+} \pi^{+} \pi^{-}, K_{S}^{0} \pi^{+} \pi^{-}, K_{S}^{0} \pi^{+} \pi^{-} \pi^{0}, K_{S}^{0} \pi^{0}$, $K^{+} K^{-}, \pi^{+} \pi^{-}$, and $K_{S}^{0} K_{S}^{0}$ channels, and $D^{+}$mesons in the $K^{-} \pi^{+} \pi^{+}, K^{-} \pi^{+} \pi^{+} \pi^{0}, K_{S}^{0} \pi^{+}, K_{S}^{0} \pi^{+} \pi^{0}, K^{+} K^{-} \pi^{+}$, $K_{S}^{0} K^{+}$, and $K_{S}^{0} \pi^{+} \pi^{+} \pi^{-}$channels. In events with multiple $D \ell^{-}$combinations, the candidate with the best $D-\ell$ vertex fit is selected. Candidate $D^{*}$ mesons are reconstructed by combining a $D$ candidate with a pion or a photon in the $D^{*+} \rightarrow D^{0} \pi^{+}, D^{*+} \rightarrow D^{+} \pi^{0}, D^{* 0} \rightarrow D^{0} \pi^{0}$, and $D^{* 0} \rightarrow$ $D^{0} \gamma$ channels. In events with multiple $D^{*} \ell^{-}$combinations, we choose the candidate with the smallest $\chi^{2}$ based on the deviations from the nominal values of the $D$ invariant mass and the invariant mass difference between the $D^{*}$ and the $D$, using the resolution measured in each mode.

We reconstruct $B_{\text {tag }}$ decays [16] in charmed hadronic modes $\bar{B} \rightarrow D Y$, where $Y$ represents a collection of hadrons, composed of $n_{1} \pi^{ \pm}+n_{2} K^{ \pm}+n_{3} K_{S}^{0}+n_{4} \pi^{0}$, where $n_{1}+n_{2}=1,3,5, n_{3} \leq 2$, and $n_{4} \leq 2$. Using $D^{0}\left(D^{+}\right)$and $D^{* 0}\left(D^{*+}\right)$ as seeds for $B^{-}\left(\bar{B}^{0}\right)$ decays, we reconstruct about 1000 different decay chains.

The kinematic consistency of a $B_{\text {tag }}$ candidate with a $B$ meson decay is evaluated using two variables: the beamenergy substituted mass $m_{\mathrm{ES}} \equiv \sqrt{s / 4-\left|p_{B}^{*}\right|^{2}}$, and the energy difference $\Delta E \equiv E_{B}^{*}-\sqrt{s} / 2$. Here, $\sqrt{s}$ is the total c.m. energy, and $p_{B}^{*}$ and $E_{B}^{*}$ denote the momentum and 
energy of the $B_{\text {tag }}$ candidate in the c.m. frame. For correctly identified $B_{\mathrm{tag}}$ decays, the $m_{\mathrm{ES}}$ distribution peaks at the $B$ meson mass, while $\Delta E$ is consistent with zero. We select $B_{\text {tag }}$ candidates in the signal region defined as $5.27 \mathrm{GeV} / c^{2}<m_{\mathrm{ES}}<5.29 \mathrm{GeV} / c^{2}$, excluding those with daughter particles in common with the charm meson or the lepton from the semileptonic $B$ decay. In the case of multiple $B_{\text {tag }}$ candidates in an event, we select the one with the smallest $|\Delta E|$ value. The $B_{\text {tag }}$ and the $D^{(*)} \ell$ candidates are required to have the correct charge-flavor correlation. We account for mixing effects in the $\bar{B}^{0}$ sample as described in Ref. [17]. Cross-feed effects, i.e., $B_{\mathrm{tag}}^{-}\left(\bar{B}_{\mathrm{tag}}^{0}\right)$ candidates erroneously reconstructed as a neutral (charged) $B$, are subtracted using estimates from the simulation.

We reconstruct $\quad B^{-} \rightarrow D^{(*)+} \pi^{-} \ell^{-} \bar{\nu}_{\ell} \quad$ and $\quad \bar{B}^{0} \rightarrow$ $D^{(*) 0} \pi^{+} \ell^{-} \bar{\nu}_{\ell}$ decays starting from the corresponding $B_{\text {tag }}+D^{(*)} \ell^{-}$combinations. We select events with only one additional reconstructed charged track, correctly matched to the $D^{(*)}$ flavor, that has not been used for the reconstruction of the $B_{\text {tag }}$, the signal $D^{(*)}$, or the lepton. $D\left(D^{*}\right)$ candidates are selected within $2 \sigma$ (1.5-2.5 $\sigma$, depending on the $D^{*}$ decay mode) of the $D$ mass $\left(D^{*}-D\right.$ mass difference), where the resolution $\sigma$ is typically around $8(1-7) \mathrm{MeV} / c^{2}$. For the $\bar{B}^{0} \rightarrow D^{(*) 0} \pi^{+} \ell^{-} \bar{\nu}_{\ell}$ decay, we additionally require the invariant mass difference $m\left(D^{0} \pi^{+}\right)-m\left(D^{0}\right)$ to be greater than $0.18 \mathrm{GeV} / c^{2}$ to veto $\bar{B}^{0} \rightarrow D^{*+} \ell^{-} \bar{\nu}_{\ell}$ events.

Semileptonic $\bar{B} \rightarrow D^{* *} \ell^{-} \bar{\nu}_{\ell}$ decays are identified by the missing mass squared in the event, $m_{\text {miss }}^{2}=\{p[\Upsilon(4 S)]-$ $\left.p\left(B_{\text {tag }}\right)-p\left(D^{(*)} \pi\right)-p(\ell)\right\}^{2}$, defined in terms of the particle four momenta. For correctly reconstructed signal events, the only missing particle is the neutrino, and $m_{\text {miss }}^{2}$ peaks at zero. Other $B$ semileptonic decays, where one particle is not reconstructed (feed-down) or is erroneously added to the charm candidate (feed-up), exhibit higher or lower values in $m_{\text {miss }}^{2}$ [7]. In feed-down cases where both a $D$ and a $D^{*}$ candidate have been reconstructed, we keep only the latter candidate.

The $m_{\text {miss }}^{2}$ selection criteria are listed in Table I. The $m_{\text {miss }}^{2}$ region between 0.2 and $1 \mathrm{GeV}^{2} / c^{4}$ for $\bar{B} \rightarrow$ $D \pi \ell^{-} \bar{\nu}_{\ell}$ events is dominated by feed-down from $\bar{B} \rightarrow$ $D^{* *}\left(\rightarrow D^{*} \pi\right) \ell^{-} \bar{\nu}_{\ell}$ semileptonic decays where the soft pion from the $D^{*}$ decay is not reconstructed. In order to retain these events, we apply an asymmetric cut on $m_{\text {miss }}^{2}$

TABLE I. $\quad m_{\text {miss }}^{2}$ selection criteria.

\begin{tabular}{cc}
\hline \hline \multicolumn{1}{c}{ Mode } & Selection Criteria \\
\hline$B^{-} \rightarrow D^{*+} \pi^{-} \ell^{-} \bar{\nu}_{\ell}$ & $-0.25<m_{\text {miss }}^{2}<0.25 \mathrm{GeV}^{2} / c^{4}$ \\
$B^{-} \rightarrow D^{+} \pi^{-} \ell^{-} \bar{\nu}_{\ell}$ & $-0.25<m_{\text {miss }}^{2}<0.8 \mathrm{GeV}^{2} / c^{4}$ \\
$\bar{B}^{0} \rightarrow D^{* 0} \pi^{+} \ell^{-} \bar{\nu}_{\ell}$ & $-0.2<m_{\text {miss }}^{2}<0.35 \mathrm{GeV}^{2} / c^{4}$ \\
$\bar{B}^{0} \rightarrow D^{0} \pi^{+} \ell^{-} \bar{\nu}_{\ell}$ & $-0.15<m_{\text {miss }}^{2}<0.85 \mathrm{GeV}^{2} / c^{4}$ \\
\hline \hline
\end{tabular}

for these modes. As a cross check, we repeat the analysis using a symmetric cut on $m_{\text {miss }}^{2}$ for each event sample, obtaining results consistent with the ones presented below.

The signal yields for the $\bar{B} \rightarrow D^{* *} \ell^{-} \bar{\nu}_{\ell}$ decays are extracted through a simultaneous unbinned maximum likelihood fit to the four $m\left(D^{(*)} \pi\right)-m\left(D^{(*)}\right)$ distributions. With the current statistics, validation studies on MC samples show that our sensitivity to nonresonant $\bar{B} \rightarrow$ $D^{(*)} \pi \ell^{-} \bar{\nu}_{\ell}$ decays is limited. Including hypotheses for these components results in a fitted contribution that is consistent with zero. Thus, we assume that these nonresonant contributions are negligible. The probability that $\bar{B} \rightarrow$ $D^{* *}\left(\rightarrow D^{*} \pi\right) \ell^{-} \bar{\nu}_{\ell}$ decays are reconstructed as $\bar{B} \rightarrow$ $D^{* *}(\rightarrow D \pi) \ell^{-} \bar{\nu}_{\ell}$ is determined with the MC simulation to be $26 \%(59 \%)$ for the $B^{-}\left(\bar{B}^{0}\right)$ sample and held fixed in the fit.

The Probability Density Functions (PDFs) for the $D^{* *}$ signal components are determined using $\mathrm{MC} \quad \bar{B} \rightarrow$ $D^{* *} \ell^{-} \bar{\nu}_{\ell}$ signal events. A convolution of a Breit-Wigner function with a Gaussian, whose resolution is determined from the simulation, is used to model the $D^{* * *}$ resonances. The $D^{* *}$ masses and widths are fixed to measured values [5]. We rely on the MC prediction for the shape of the combinatorial and continuum background. A nonparametric KEYS function [18] is used to model this component for the $D^{*} \pi \ell^{-} \bar{\nu}_{\ell}$ sample, while for the $D \pi \ell^{-} \bar{\nu}_{\ell}$ sample, we use the convolution of an exponential with a Gaussian to model the tail from virtual $D^{*}$ mesons. The combinatorial and continuum background yields are estimated from data. We fit the hadronic $B_{\mathrm{tag}} m_{\mathrm{ES}}$ distributions for $\bar{B} \rightarrow$ $D^{* *} \ell^{-} \bar{\nu}_{\ell}$ events as described in [7], and we obtain the number of background events from the integral of the background function in the $m_{\mathrm{ES}}$ signal region.

Table II summarizes the results from two fits: one in which we fit the charged and neutral $B$ samples separately, and one in which we impose the isospin constraints $\mathcal{B}\left(B^{-} \rightarrow D^{* *} \ell^{-} \bar{\nu}_{\ell}\right) / \mathcal{B}\left(\bar{B}^{0} \rightarrow D^{* *} \ell^{-} \bar{\nu}_{\ell}\right)=\tau_{B^{-}} / \tau_{\bar{B}^{0}}$. The latter fit yields a significance greater than 5 standard deviations for all four $D^{* *}$ states including systematic uncertainties. The results of this fit are shown in Fig. 1.

The $D_{2}^{*}$ contributes to both the $D \pi$ and the $D^{*} \pi$ samples. In the nominal fit, we fix the ratio $\mathcal{B}\left(D_{2}^{*} \rightarrow\right.$ $D \pi) / \mathcal{B}\left(D_{2}^{*} \rightarrow D^{*} \pi\right)$ to 2.2 [5]. When we allow this ratio to float, we obtain $1.9 \pm 0.6$.

To reduce systematic uncertainties, we measure the ratios of the $\mathcal{B}\left(\bar{B} \rightarrow D^{* *} \ell^{-} \bar{\nu}_{\ell}\right)$ branching fractions to the inclusive $\bar{B}^{0}$ and $B^{-}$semileptonic branching fractions. A sample of $\bar{B} \rightarrow X \ell^{-} \bar{\nu}_{\ell}$ events is selected by identifying a charged lepton with $p_{\ell}^{*}>0.6 \mathrm{GeV} / c$ and the correct charge correlation with the $B_{\text {tag }}$ candidate. In the case of multiple $B_{\text {tag }}$ candidates in an event, we select the one reconstructed in the decay channel with the highest purity, defined as the fraction of signal events in the $m_{\mathrm{ES}}$ signal region. Background components that peak in the $m_{\mathrm{ES}}$ signal region include cascade $B$ meson decays (i.e., the 
TABLE II. Results from the fits to data: the $\bar{B} \rightarrow D^{* *} \ell^{-} \bar{\nu}_{\ell}$ signal yield, the corresponding reconstruction efficiency, the product of branching fractions, where the first error is statistical and the second systematic. For the $\bar{B} \rightarrow D_{2}^{*} \ell^{-} \bar{\nu}_{\ell}$ decay, we report yields and product of branching fractions for the $D_{2}^{*} \rightarrow D \pi$ decay mode. For the isospin-constrained results (last two columns), the $B^{-}$branching fraction products are reported. The statistical significances, $S_{\text {stat }}$, are obtained by computing the difference in the log likelihood between the nominal fit and the fit in which we fix the different signal components to 0 . The significances including the systematic uncertainty, $S_{\text {tot }}$, are obtained by rescaling the statistical significances by $\sigma_{\text {stat }} / \sqrt{\sigma_{\text {stat }}^{2}+\sigma_{\text {syst }}^{2}}$.

\begin{tabular}{lrrclll}
\hline \hline Decay Mode & \multicolumn{1}{c}{ Yield } & $\epsilon_{\text {sig }}\left(\times 10^{-4}\right)$ & $\mathcal{B}\left(\bar{B} \rightarrow D^{* *} \ell^{-} \bar{\nu}_{\ell}\right) \times \mathcal{B}\left(D^{* *} \rightarrow D^{(*)} \pi^{ \pm}\right) \%$ & $S_{\text {tot }}\left(S_{\text {stat }}\right)$ & $\mathcal{B} \%$ & $S_{\text {tot }}\left(S_{\text {sitat }}\right)$ \\
\hline$B^{-} \rightarrow D_{1}^{0} \ell^{-} \bar{\nu}_{\ell}$ & $165 \pm 18$ & 1.24 & $0.29 \pm 0.03 \pm 0.03$ & $9.9(12.7)$ & $0.29 \pm 0.03 \pm 0.03$ & $10.7(15.2)$ \\
$B^{-} \rightarrow D_{2}^{* 0} \ell^{-} \bar{\nu}_{\ell}$ & $97 \pm 16$ & 1.44 & $0.15 \pm 0.02 \pm 0.02$ & $5.2(7.3)$ & $0.12 \pm 0.02 \pm 0.02$ & $5.3(7.4)$ \\
$B^{-} \rightarrow D_{1}^{\prime 0} \ell^{-} \bar{\nu}_{\ell}$ & $142 \pm 21$ & 1.13 & $0.27 \pm 0.04 \pm 0.05$ & $5.4(8.0)$ & $0.30 \pm 0.03 \pm 0.04$ & $6.4(10.0)$ \\
$B^{-} \rightarrow D_{0}^{* 0} \ell^{-} \bar{\nu}_{\ell}$ & $137 \pm 26$ & 1.15 & $0.26 \pm 0.05 \pm 0.04$ & $4.5(5.8)$ & $0.32 \pm 0.04 \pm 0.04$ & $6.1(8.3)$ \\
$\bar{B}^{0} \rightarrow D_{1}^{+} \ell^{-} \bar{\nu}_{\ell}$ & $88 \pm 14$ & 0.70 & $0.27 \pm 0.04 \pm 0.03$ & $7.0(8.4)$ & & \\
$\bar{B}^{0} \rightarrow D_{2}^{*+} \ell^{-} \bar{\nu}_{\ell}$ & $29 \pm 13$ & 0.91 & $0.07 \pm 0.03 \pm 0.02(<0.12 @ 90 \% \mathrm{CL})$ & $2.0(2.5)$ & & \\
$\bar{B}^{0} \rightarrow D_{1}^{\prime+} \ell^{-} \bar{\nu}_{\ell}$ & $86 \pm 18$ & 0.60 & $0.31 \pm 0.07 \pm 0.05$ & $4.6(5.8)$ & & \\
$\bar{B}^{0} \rightarrow D_{0}^{*+} \ell^{-} \bar{\nu}_{\ell}$ & $142 \pm 26$ & 0.70 & $0.44 \pm 0.08 \pm 0.06$ & $4.7(6.0)$ & & \\
\hline \hline
\end{tabular}

lepton does not come directly from the $B$ ) and hadronic decays, and are subtracted using the corresponding MC predictions.

The total yield for the inclusive $\bar{B} \rightarrow X \ell^{-} \bar{\nu}_{\ell}$ decays is obtained from a maximum likelihood fit to the $m_{\mathrm{ES}}$ distribution of the $B_{\text {tag }}$ candidates, as described in [7]. The fit yields $198897 \pm 1578$ events for the $B^{-} \rightarrow X \ell^{-} \bar{\nu}_{\ell}$ sample and $120168 \pm 1036$ events for the $\bar{B}^{0} \rightarrow X \ell^{-} \bar{\nu}_{\ell}$ sample.

The ratios $\mathcal{B}\left(\bar{B} \rightarrow D^{* *} \ell^{-} \bar{\nu}_{\ell}\right) / \mathcal{B}\left(\bar{B} \rightarrow X \ell^{-} \bar{\nu}_{\ell}\right)=$ $\left(N_{\text {sig }} / \epsilon_{\text {sig }}\right)\left(\epsilon_{\mathrm{sl}} / N_{\mathrm{sl}}\right)$ are obtained by correcting the signal yields for the reconstruction efficiencies (estimated from $B \bar{B}$ MC events). Here, $N_{\text {sig }}$ is the number of $\bar{B} \rightarrow D^{* *} \ell^{-} \bar{\nu}_{\ell}$ signal events, reported in Table II together with the corresponding reconstruction efficiencies $\epsilon_{\mathrm{sig}}, N_{\mathrm{sl}}$ is the $\bar{B} \rightarrow$ $X \ell^{-} \bar{\nu}_{\ell}$ signal yield, and $\epsilon_{\mathrm{sl}}$ is the corresponding reconstruction efficiency including the $B_{\text {tag }}$ reconstruction, equal to $0.39 \%$ and $0.25 \%$ for the $B^{-} \rightarrow X \ell^{-} \bar{\nu}_{\ell}$ and $\bar{B}^{0} \rightarrow$ $X \ell^{-} \bar{\nu}_{\ell}$ decays, respectively. The absolute branching fractions $\mathcal{B}\left(\bar{B} \rightarrow D^{* * *} \ell^{-} \bar{\nu}_{\ell}\right)$ are then determined using the semileptonic branching fraction $\mathcal{B}\left(\bar{B} \rightarrow X \ell^{-} \bar{\nu}_{\ell}\right)=$ $(10.78 \pm 0.18) \%$ and the ratio of the $\bar{B}^{0}$ and the $B^{-}$lifetimes $\tau_{B^{-}} / \tau_{\bar{B}^{0}}=1.071 \pm 0.009$ [5].

Numerous sources of systematic uncertainties have been investigated. The largest uncertainty is due to the determination of the $\bar{B} \rightarrow D^{* *} \ell^{-} \bar{\nu}_{\ell}$ signal yields (resulting in 5.5$17.0 \%$ relative systematic uncertainty depending on the $D^{* *}$ state). This uncertainty is estimated using ensembles of fits to the data in which the input parameters are varied within the known uncertainties in the PDF parameterization $(0.2-8.7 \%)$, the shape and yield of the combinatorial and continuum background $(0.2-10.4 \%)$, the modeling of the broad $D^{* *}$ states $(4.5-13.8 \%)$, and the $D^{*}$ feed-down rate $(0.5-4.0 \%)$. We check that the combinatorial and continuum background shape is well reproduced by the simulation by verifying that the MC samples of right-sign and wrong-sign $D^{(*)} \pi$ combinations have similar shapes, and that the wrong-sign distribution in the data agrees well with that in the simulation. We observe an excess of events in the low invariant mass difference region in the four samples that is not accounted for by the background PDF. We study $\bar{B} \rightarrow D^{(*)} n \pi \ell^{-} \bar{\nu}_{\ell}(n>1)$ decays, that are not included in our standard MC simulation, as a possible source of this excess. We use different MC models for these decays, and, assuming $\mathcal{B}\left(\bar{B} \rightarrow D^{(*)} n \pi \ell^{-} \bar{\nu}_{\ell}\right)=(1 \pm$ $1) \%$, we find that they account for about $30 \%$ of the observed excess. We determine the systematic uncertainty $(0.1-3.2 \%)$, included in the yield uncertainty, by repeating the fit using the different models and varying this background yield within the assumed branching fraction error. The uncertainties due to the detector simulation are determined by varying, within bounds given by data control samples, the charged track reconstruction efficiency (1.3$2.0 \%)$, the photon reconstruction efficiency $(0.2-4.8 \%)$, the lepton identification efficiency (1.2-1.6\%), and the reconstruction efficiency for low momentum charged $(1.2 \%)$ and neutral pions (1.3\%). We use an HQET model [8] to test the model dependence of the $\bar{B} \rightarrow D^{* *} \ell^{-} \bar{\nu}_{\ell}$ simulation $(0.8-2.5 \%)$. We include the uncertainty on the branching fractions of the reconstructed $D$ and $D^{*}$ modes (3.0-4.5\%), and on the absolute branching fraction $\mathcal{B}\left(\bar{B} \rightarrow X \ell^{-} \bar{\nu}_{\ell}\right)$ used for the normalization (1.9\%). We also include a systematic uncertainty due to differences in the efficiency of the $B_{\text {tag }}$ selection in the exclusive selection of $\bar{B} \rightarrow$ $D^{* *} \ell^{-} \bar{\nu}_{\ell}$ decays and the inclusive $\bar{B} \rightarrow X \ell^{-} \bar{\nu}_{\ell}$ reconstruction $(4.0-5.6 \%)$.

In conclusion, we report the simultaneous observation of $\bar{B} \rightarrow D^{* *} \ell^{-} \bar{\nu}_{\ell}$ decays into the four $D^{* *}$ states predicted by HQS. The measured branching fractions are reported in Table II. We find results consistent with Ref. [7] for the sum of the different $D^{* *}$ branching fractions. The rate for 


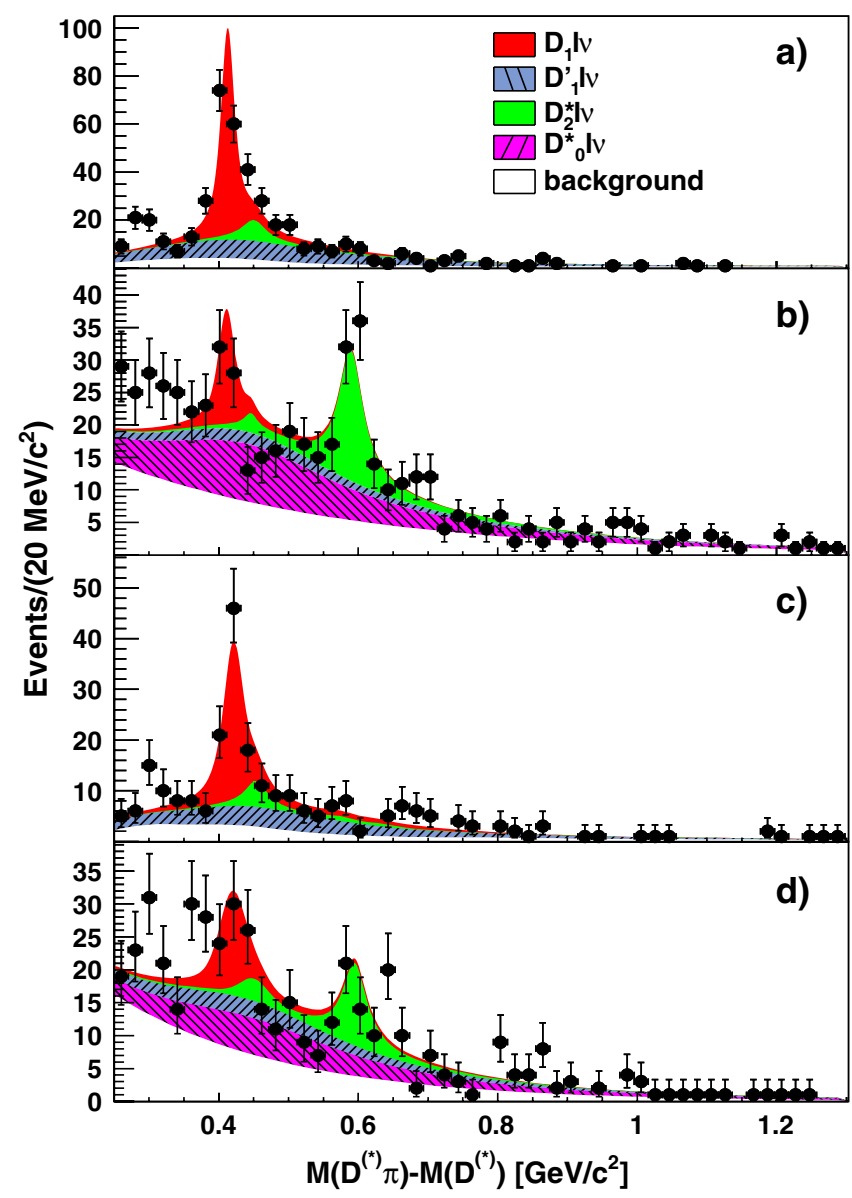

FIG. 1 (color online). Fit to the $m\left(D^{(*)} \pi\right)-m\left(D^{(*)}\right)$ distribution for (a) $B^{-} \rightarrow D^{*+} \pi^{-} \ell^{-} \bar{\nu}_{\ell}$, (b) $B^{-} \rightarrow D^{+} \pi^{-} \ell^{-} \bar{\nu}_{\ell}$, (c) $\bar{B}^{0} \rightarrow D^{* 0} \pi^{+} \ell^{-} \bar{\nu}_{\ell}$, and (d) $\bar{B}^{0} \rightarrow D^{0} \pi^{+} \ell^{-} \bar{\nu}_{\ell}$ : the data (points with error bars) are compared to the results of the overall fit (sum of the solid distributions). The PDFs for the different fit components are stacked and shown in different colors.

the $D^{* *}$ narrow states is in good agreement with recent measurements [19]; the one for the broad states is in agreement with DELPHI [11] but does not agree with the $D_{1}^{\prime}$ limit of Belle [10]. The rate for the broad states is found to be large. If these broad states are indeed due to $\bar{B} \rightarrow$ $D_{1}^{\prime} \ell^{-} \bar{\nu}_{\ell}$ and $\bar{B} \rightarrow D_{0}^{*} \ell^{-} \bar{\nu}_{\ell}$ decays, this is in conflict with the expectations from QCD sum rules.

We are grateful for the excellent luminosity and machine conditions provided by our PEP-II colleagues, and for the substantial dedicated effort from the computing organizations that support $B A B A R$. The collaborating institutions wish to thank SLAC for its support and kind hospitality. This work is supported by DOE and NSF (USA), NSERC
(Canada), CEA and CNRS-IN2P3 (France), BMBF and DFG (Germany), INFN (Italy), FOM (The Netherlands), NFR (Norway), MIST (Russia), MEC (Spain), and STFC (United Kingdom). Individuals have received support from the Marie Curie EIF (European Union) and the A. P. Sloan Foundation.

* Deceased

${ }^{\dagger}$ Now at Temple University, Philadelphia, PA 19122, USA

${ }^{*}$ Now at Tel Aviv University, Tel Aviv, 69978, Israel

${ }^{\S}$ Also with Università di Perugia, Dipartimento di Fisica, Perugia, Italy

"Also with Università di Roma La Sapienza, I-00185 Roma, Italy

IINow at University of South Alabama, Mobile, AL 36688, USA

**Also with Università di Sassari, Sassari, Italy

[1] M. Kobayashi and T. Maskawa, Prog. Theor. Phys. 49, 652 (1973).

[2] The charge conjugate state is always implied unless stated otherwise.

[3] B. Aubert et al. (BABAR Collab.), Phys. Rev. D 77, 032002 (2008).

[4] N. Isgur and M. B. Wise, Phys. Rev. Lett. 66, 1130 (1991).

[5] W.-M. Yao et al. (Particle Data Group), J. Phys. G 33, 1 (2006).

[6] B. Aubert et al. (BABAR Collab.), Phys. Rev. D 76, 051101 (2007).

[7] B. Aubert et al. (BABAR Collab.), Phys. Rev. Lett. 100, 151802 (2008).

[8] A. K. Leibovich, Z. Ligeti, I. W. Stewart, and M. B. Wise, Phys. Rev. D 57, 308 (1998).

[9] N. Uraltsev, Phys. Lett. B 501, 86 (2001).

[10] D. Liventsev et al. (Belle Collab.), Phys. Rev. D 77, 091503 (2008).

[11] J. Abdallah et al. (DELPHI Collab.), Eur. Phys. J. C 45, 35 (2006).

[12] B. Aubert et al. (BABAR Collab.), Nucl. Instrum. Methods Phys. Res., Sect. A 479, 1 (2002).

[13] S. Agostinelli et al., Nucl. Instrum. Methods Phys. Res., Sect. A 506, 250 (2003).

[14] D. Scora and N. Isgur, Phys. Rev. D 52, 2783 (1995); See also N. Isgur et al., ibid. 39, 799 (1989).

[15] J. L. Goity and W. Roberts, Phys. Rev. D 51, 3459 (1995).

[16] B. Aubert et al. (BABAR Collab.), Phys. Rev. Lett. 92, 071802 (2004).

[17] B. Aubert et al. (BABAR Collab.), Phys. Rev. D 69, 111104 (2004).

[18] K. Cranmer, Comput. Phys. Commun. 136, 198 (2001).

[19] V. Abazov et al. (D0 Collab.), Phys. Rev. Lett. 95, 171803 (2005). 